\title{
Sustained Remission Improves Physical Function in Patients with Established Rheumatoid Arthritis, and Should Be a Treatment Goal: A Prospective Observational Cohort Study from Southern Sweden
}

\author{
Jon Thorkell Einarsson, Pierre Geborek, Tore Saxne, Lars Erik Kristensen, \\ and Meliha C. Kapetanovic
}

\begin{abstract}
Objective. It has been proposed that remission should be maintained throughout the course of rheumatoid arthritis (RA); however, the evidence supporting this is limited. Physical function measured by the Health Assessment Questionnaire (HAQ) is a major outcome in RA, and HAQ is shown to be one of the strongest predictors of longterm outcomes. The purpose of this study was to investigate the physical function over a long time in patients with RA who achieved sustained remission (SR) compared with that of patients occasionally achieving remission [non-sustained remission (NSR)].

Methods. Patients with RA treated with antitumor necrosis factor and included in the South Swedish Arthritis Treatment Group register were eligible for this study. We identified patients with a Disease Activity Score at 28 joints (DAS28) $<2.6$ or Simplified Disease Activity Index (SDAI) $\leq 3.3$ at some point and those who achieved SR, i.e., remission during consecutive visits for at least 6 months. The course of functional status was assessed using the HAQ at each visit.

Results. Of the 2416 patients, 1177 (48.7\%) reached DAS28 remission at some point. SR was achieved by $382(15.8 \%)$ for the DAS28 and $186(7.7 \%)$ for the SDAI criteria. Comparing the SR and NSR groups, HAQ improved during the first 12 months in the DAS28 remission. HAQ continued to improve relatively as long as SR was maintained. A higher proportion of patients in SR reached full physical function.

Conclusion. In patients with established RA, physical function measured by the HAQ improves in patients reaching SR compared with patients who only occasionally reach remission. The improvement continues while in remission, which supports that maintaining remission should be a treatment goal. (First Release April 1 2016; J Rheumatol 2016;43:1017-23; doi:10.3899/ jrheum.150995)
\end{abstract}

Key Indexing Terms: RHEUMATOID ARTHRITIS DISEASE ACTIVITY SCORE HEALTH ASSESSMENT QUESTIONNAIRE

REMISSION BIOLOGICAL THERAPY

Rheumatoid arthritis (RA) is a chronic inflammatory disease associated with substantial pain, joint damage, functional impairment, and disability ${ }^{1}$. Modern antirheumatic treatment, including traditional disease-modifying antirheumatic drugs (DMARD) and biologic remedies with different modes of actions, have been proven to be effective in improving signs and symptoms of inflammation ${ }^{2}$. Remission is increasingly becoming a treatment goal in patients with RA since the introduction of the "treat to target"3 concept. It has also been proposed that remission should be maintained throughout the course of the disease ${ }^{3}$; however, the evidence supporting this is still limited ${ }^{3}$.
From the Department of Clinical Sciences Lund, Section of Rheumatology, Lund University, Skåne University Hospital, Lund, Sweden; The Parker Institute, Department of Rheumatology, Bispebjerg and Frederiksberg Hospital, the Capital Region of Copenhagen, Copenhagen, Denmark. Supported by grants from the Swedish Rheumatism Association, the Swedish Research Council, the Medical Faculty of Lund University, the Alfred Österlund Foundation, the Crafoord Foundation, the Greta and Johan Kock Foundation, King Gustaf V's 80-Year Foundation, the Oak foundation, and Lund University Hospital.

J.T. Einarsson, MD, Department of Clinical Sciences Lund, Section of Rheumatology, Lund University, Skåne University Hospital; P. Geborek, $M D, P h D$, Department of Clinical Sciences Lund, Section of

Rheumatology, Lund University, Skåne University Hospital; T. Saxne, MD,
PhD, Professor, Department of Clinical Sciences Lund, Section of Rheumatology, Lund University, Skåne University Hospital; L.E. Kristensen, MD, PhD, Department of Clinical Sciences Lund, Section of Rheumatology, Lund University, Skåne University Hospital, and The Parker Institute, Department of Rheumatology, Bispebjerg and Frederiksberg Hospital, the Capital Region of Copenhagen; M.C. Kapetanovic, MD, PhD, Department of Clinical Sciences Lund, Section of Rheumatology, Lund University, Skåne University Hospital.

Address correspondence to Dr. J.T. Einarsson, Department of Clinical Sciences Lund, Section of Rheumatology, Skånes University Hospital. Lund University, Kioskgatan 3, SE-221 85 Lund, Sweden. E-mail:jonthorkell@gmail.com

Accepted for publication February 17, 2016.

Personal non-commercial use only. The Journal of Rheumatology Copyright (C) 2016. All rights reserved. 
Joint damage is the hallmark of RA, and there is limited evidence that sustained remission (SR) by itself halts progression of joint damage and that any increase in disease activity may reignite the destructive progress ${ }^{4}$. Functional status measured by the Health Assessment Questionnaire (HAQ) is a major outcome in RA. The HAQ has been shown to be among the strongest predictors of longterm outcomes, including work disability and mortality, even when compared to radiographic progression ${ }^{5,6}$.

No universally accepted remission criteria exist for remission in established RA, let alone for SR. Remission as defined by the Disease Activity Score at 28 joints (DAS28) is not regarded as sufficiently stringent ${ }^{7}$. Instead, DAS28 < 2.6 may represent a minimal disease activity rather than remission ${ }^{8}$. However, it is still the most widely used criterion for remission in patients with established disease. The newer American College of Rheumatology (ACR)/European League Against Rheumatism (EULAR) criteria and the criteria based on the Clinical Disease Activity Index (CDAI) and the Simplified Disease Activity Index (SDAI) are more stringent in defining remission ${ }^{9}$. That means that when these definitions are applied, fewer patients reach remission, but in turn have a better functional status.

The purposes of our study were to investigate the effect of SR on physical function measured by HAQ in patients with established RA and whether SR was associated with better physical function compared with occasionally reaching remission. We also aimed to compare different types of remission criteria and their possible effect on this outcome.

\section{MATERIALS AND METHODS}

Data were retrieved from the South Swedish Arthritis Treatment Group (SSATG) register, a large, prospective, observational study cohort involving 11 rheumatology clinics. The registry contained detailed disease severity characteristics at baseline and at followup for patients treated with biologic drugs dating from $1999^{10}$. Several validation studies have proved it to be population-based for patients with RA, with coverage of pharmacy sales of $>90 \%{ }^{11,12}$. In Sweden, the government is the only buyer of healthcare, and during the study period, the treating physician had no restrictions for selecting biologic therapy and did not need to taper treatment when remission was achieved.

All patients with RA were eligible for our study if they had been diagnosed according to the clinical judgment of the treating physician and had started antitumor necrosis factor (anti-TNF) treatment during the period from March 1999 through December 2009. No formal level of disease activity was required; however, the patients should have received at least 1 DMARD previously without acceptable response. A previous review of patients included in this cohort showed that $98 \%$ of the patients fulfilled the 1987 ACR classification criteria for RA ${ }^{13}$. The course of functional status was assessed using HAQ at each visit. Composite indices, such as the CDAI, SDAI, and DAS28, and the DAS-erythrocyte sedimentation rate were calculated. Information on study protocol and frequency of variables collected is in the supplementary data (available from the authors on request).

The registry was searched for individual treatments fulfilling the DAS28, SDAI, and CDAI remission criteria with cutpoints at $<2.6,3.3$, and 2.8, respectively ${ }^{14}$. One researcher (JTE) scrutinized every treatment case by case, ensuring true remission. Two remission groups were defined. Patients who fulfilled the remission criteria on at least 2 consecutive occasions and for at least 6 months after $\geq 3$ months of treatment initiation were categorized as having SR. Patients who fulfilled the remission criteria at only 1 timepoint or occasionally but not sustained over time were defined as having non-sustained remission (NSR). NSR started when the patient reached remission for the first time. Remission time was defined as time between first visit fulfilling the remission criteria and subsequent first visit with higher disease activity. After escape from SR, patients continued to be in another group (SR and escape).

Only the first registered treatment per patient was analyzed, i.e., individual patients contributed only 1 SR treatment. Followup data were gathered at treatment start and again from remission start at every visit as long as the patient continued treatment with the same medication. Data were collected through to June 2010 or until treatment termination. No data imputation was done.

Statistical analysis. Demographic, patient, and disease characteristics at the start of anti-TNF therapy were calculated in all patients and also after stratification according to reaching remission, i.e., NSR and SR (Table 1). Descriptive statistics for categorical variables are presented as frequencies or percentages, and differences between groups were analyzed using the chi-square test. Continuous variables are presented as means, and differences were analyzed using the Mann-Whitney U test.

Mean HAQ was calculated for each patient at each year so that for a given year, a patient contributed only 1 mean HAQ score. When a patient escaped remission, only the HAQ scores collected earlier that year were used. The mean HAQ scores for each followup year and remission group were calculated, as well as for baseline values (remission start). Results are given as 95\% CI. Number of patients reaching 0 in HAQ score (full physical function, HAQ = 0) are presented as a fraction of the group with 95\% CI. For statistical comparison, we calculate the change in HAQ ( $\triangle \mathrm{HAQ})$ from remission start at each year.

SPSS Statistics version 20.0 and Sigma Plot version 13.0 were used to conduct the analyses.

\section{RESULTS}

Between March 1999 and December 2009, 2416 patients with RA initiated anti-TNF therapy. Mean followup time from treatment start to either treatment stop or study stop was 40 months (range $0-149$ ).

Of all 2416 patients, $1177(48.7 \%)$ reached DAS28 remission at some point. SR fulfillment was $382(15.8 \%)$ for the DAS28, $186(7.7 \%)$ SDAI, and 177 (7.3\%) CDAI criteria (Figure 1). On average, 12.2 HAQ scores (range 2-81) were documented for the SR group compared with 10.2 (range 1-100) for the NSR group within the time frame of our study. Patient characteristics at baseline are presented in Table 1. Compared with NSR patients, those who reached SR were more likely to take methotrexate at baseline, have a shorter disease duration, and have a lower HAQ at both baseline and remission start. Patients reaching DAS28 SR had lower disease activity at baseline than NSR patients (Table 1). In the following presentation of results, we compared patients in SR with NSR, i.e., improvement or worsening in relation to the other group.

Measure of function over time. At remission start, patients who reached sustained DAS28 remission had a lower mean HAQ (mean $0.46,95 \%$ CI $0.41-0.51$ ) than NSR patients who reached only point remission or occasional remission $(0.73$, 95\% CI 0.68-0.78; Figure 2A); the same applied for SDAI (Figure 2B) and/or CDAI remission (Supplementary Figure 2 , available from the authors on request). When comparing

Personal non-commercial use only. The Journal of Rheumatology Copyright $\odot$ (2016. All rights reserved 
Table 1. Demographic, disease, and patient characteristics at the start of anti-TNF therapy in all patients and also after stratification according to having reached SR and NSR. Values are means unless otherwise specified.

\begin{tabular}{|c|c|c|c|c|c|c|c|}
\hline \multirow[t]{2}{*}{ Demographics } & \multirow[t]{2}{*}{ All } & \multicolumn{3}{|c|}{ DAS28 } & \multicolumn{3}{|c|}{ SDAI } \\
\hline & & NSR & $\mathrm{SR}$ & $\mathrm{p}$ & NSR & $\mathrm{SR}$ & $\mathrm{p}$ \\
\hline $\mathrm{n}$ & 2416 & 795 & 382 & & 472 & 186 & \\
\hline Age, yrs & 56.0 & 54.4 & 52.4 & 0.023 & 53.6 & 52.7 & 0.28 \\
\hline Female sex, $\%$ & 77 & 76 & 66 & $<0.001$ & 73 & 67 & 0.13 \\
\hline Disease duration, yrs & 11.8 & 11.5 & 10.1 & 0.048 & 10.8 & 8.6 & 0.004 \\
\hline SJC28 & 9.2 & 9.0 & 8.9 & 0.92 & 9.4 & 9.4 & 0.74 \\
\hline DAS28,0-9.4 & 5.5 & 5.3 & 4.9 & $<0.001$ & 5.2 & 5.1 & 0.60 \\
\hline SDAI, 0-86 & 32.9 & 32.0 & 29.1 & $<0.001$ & 31.1 & 30.3 & 0.56 \\
\hline CDAI, $0-76$ & 30.1 & 29.4 & 26.6 & $<0.001$ & 28.5 & 27.4 & 0.37 \\
\hline Previous DMARD, $\mathrm{n}$ & 3.0 & 3.1 & 2.6 & 0.05 & 2.9 & 2.5 & 0.38 \\
\hline MTX, yes/no, \% & 61 & 64 & 77 & $<0.001$ & 64 & 80 & $<0.001$ \\
\hline Prednisolone, yes/no, \% & 59 & 61 & 56 & 0.14 & 54 & 58 & 0.37 \\
\hline HAQ, $0-3$ & 1.3 & 1.3 & 1.0 & $<0.001$ & 1.2 & 1.0 & 0.008 \\
\hline HAQ remission start, $0-3$ & - & 0.73 & 0.46 & $<0.001$ & 0.53 & 0.37 & $<0.001$ \\
\hline $\mathrm{HAQ}=0, \%$ & 1.4 & 1.4 & 4.4 & 0.002 & 2.8 & 2.7 & 0.35 \\
\hline$\Delta$ HAQ yrs $0-5$ & & 0.20 & -0.04 & $<0.05$ & 0.23 & -0.13 & $<0.05$ \\
\hline \pm & & \pm & \pm & & \pm & \pm & \\
\hline $95 \% \mathrm{CI}$ of mean & & 0.09 & 0.11 & & 0.11 & 0.12 & \\
\hline
\end{tabular}

Anti-TNF: anti-tumor necrosis factor; SR: sustained remission; NSR: non-sustained remission; DAS28: Disease Activity Score at 28 joints; SDAI: Simplified Disease Activity Index; SJC28: swollen joint count at 28 joints; CDAI: Clinical Disease Activity Index; DMARD: disease-modifying antirheumatic drugs; MTX: methotrexate; HAQ: Health Assessment Questionnaire; HAQ = 0: full physical function, score null on the HAQ; $\triangle \mathrm{HAQ}$ yrs 0-5: the mean difference in HAQ between remission start and end of Year 5 in SR with 95\% CI of the mean.

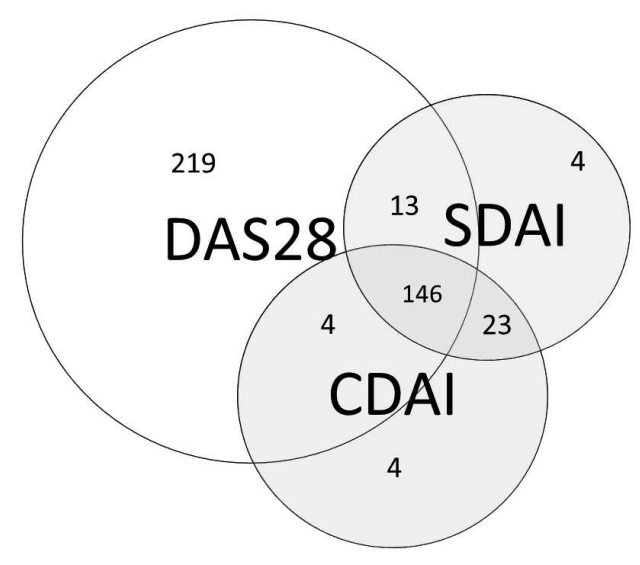

Figure 1. A Venn diagram showing the number of patients reaching SR according to different clinical indices. SR fulfillment was $382(15.8 \%)$ for the DAS28, $186(7.7 \%)$ the SDAI, and 177 (7.3\%) for the CDAI criteria. SR: sustained remission; DAS28: Disease Activity Score based on 28 joints; CDAI: Clinical Disease Activity Index; SDAI: Simplified Disease Activity Index.

these groups, the mean HAQ diverged in the first 12 months in DAS28 remission (Figure 2C) and during the first 24 months for patients in SDAI (Figure 2B) and CDAI remission (Supplementary Figure 2, available from the authors on request). The difference in HAQ continued to increase relatively as long as SR was maintained (Figure 2A, Figure 2B, Figure 2C). After 5 years in DAS28 remission, mean HAQ improved by 0.04 , but worsened by 0.20 in NSR patients (Figure 2, Table 1). After escape from SR, the relative improvement in HAQ remained (Figure 2A). To compensate for multiple comparisons, a figure with $99 \%$ CI was also calculated with similar results (Supplementary Figure 3, available from the authors on request). The same trend was seen with sustained CDAI and SDAI remission (Table 1, Figure 2B), and there was no difference in the mean HAQ between different remission criteria (Figure 2D).

Women in SR have a mean HAQ of 0.52 at remission start compared with 0.79 in the NSR group. After 2 years in remission, the mean HAQ in these groups diverged and was significantly lower in the SR group (Figure 3A). In comparison, male patients in the SR group had a lower HAQ at remission start, 0.35 and 0.56 in SR and NSR patients, respectively. Male patients in the SR group had a stable HAQ that did not improve compared with the NSR group (Figure 3B).

Full physical function. At treatment start, most patients had some level of disability (Table 1). At DAS28 remission start, $28.7 \%$ of patients who entered SR had full physical function compared with $15.8 \%$ of NSR patients. After 4 years of SR, $40.0 \%$ had full physical function compared with $10.4 \%$ of NSR patients, the difference being significant from the second year of remission (Figure 4A). A higher fraction of patients in SDAI remission had full physical function at remission start $(41 \%)$ than those in DAS28 remission. After 6 years in SDAI remission, $60 \%$ had full physical function compared with $43 \%$ in DAS28 remission (nonsignificant; Figure 4B).

Personal non-commercial use only. The Journal of Rheumatology Copyright $\odot$ 2016. All rights reserved. 

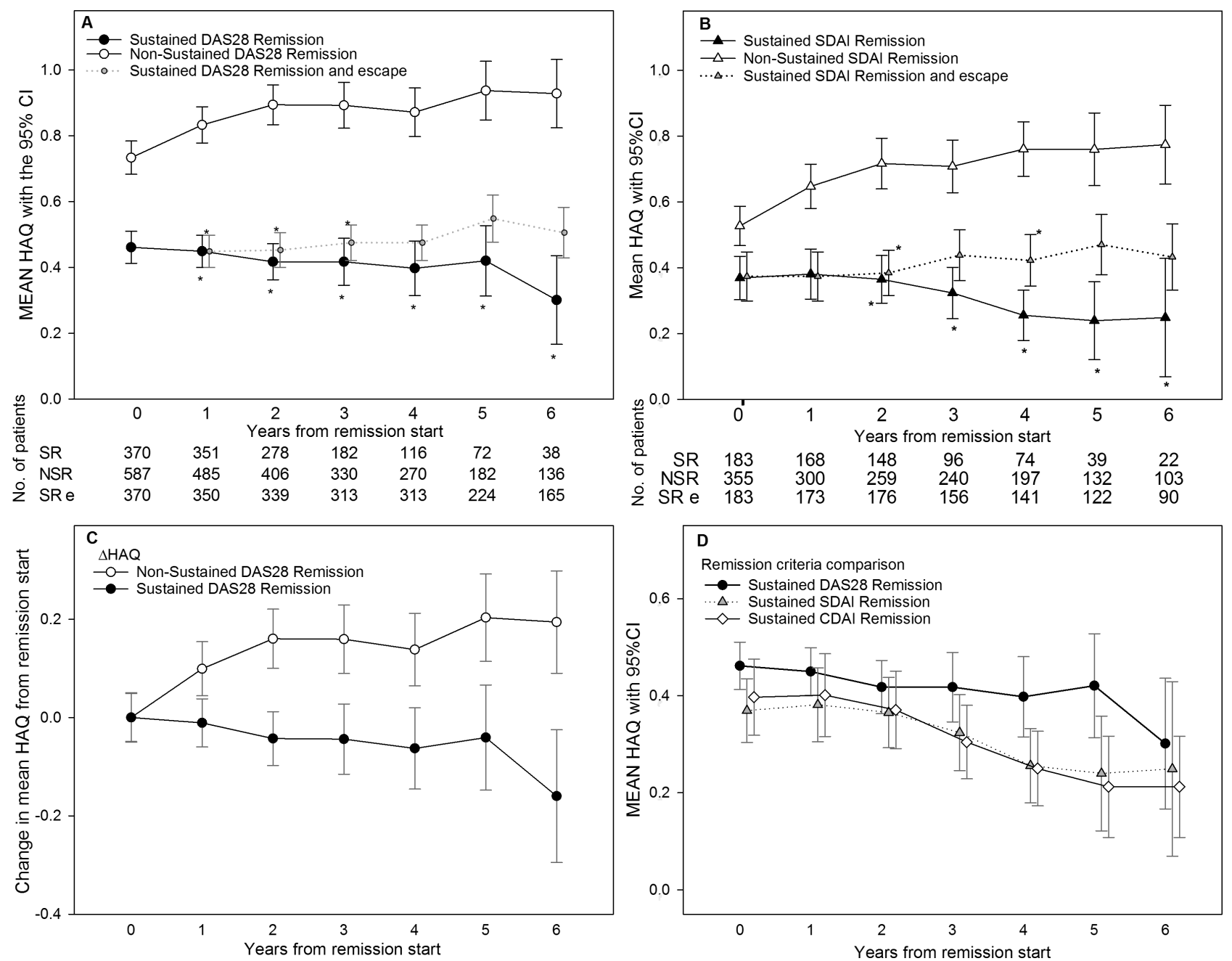

Figure 2. Course of HAQ in sustained remission. Year 0 is at start of SR or first remission for the NSR patient. After that, each point represents the mean HAQ during the year. (A) Sustained DAS28 remission defined as DAS28 $<2.6$ on at least 2 consecutive occasions and for at least 6 months. Patients fulfilling the remission criteria at only 1 timepoint or occasionally were defined as in NSR. When patients in SR escape from remission, they move from SR but are shown in SR and escape (SR e; small grey circles). The table below the figure shows the number of patients with available HAQ each year. (B) Sustained SDAI remission defined as SDAI 3.3 or less on at least 2 consecutive occasions and for at least 6 months. Other groups defined as in Figure 2A. The table below the figure shows the number of patients with available HAQ each year. (C) Mean change in HAQ during sustained DAS28 remission. Same groups as in Figure $2 \mathrm{~A}$, but now only the change in HAQ ( $\triangle \mathrm{HAQ}$ ) from remission start is shown. (D) Course of mean HAQ in sustained DAS28, CDAI, and SDAI remission compared, and no control group are shown. No differences between different remission criteria. * P < 0.05. HAQ: Health Assessment Questionnaire; SR: sustained remission; NSR: non-sustained remission; DAS28: Disease Activity Score based on 28 joints; SDAI: Simplified Disease Activity Index; CDAI: Clinical Disease Activity Index.

\section{DISCUSSION}

In our observational study of patients with established RA, physical function measured by HAQ improves in patients in SR compared with patients who reach only point remission or occasional remission. This was observed regardless of which remission criteria were used. The results support maintaining remission as a major treatment goal in established RA.

Our results are in line with earlier studies comparing remission and low disease activity (LDA) using different composite indices ${ }^{15}$. We also confirmed that DAS28 classified more patients into remission than the SDAI and CDAI criteria ${ }^{9,16}$. Few studies have examined the different outcomes of patients in clinical remission compared with LDA. An Austrian study focusing on this found that patients in SDAI or CDAI, but not DAS28, remission had significantly lower HAQ than patients with LDA and that going from $\mathrm{LDA}$ to remission resulted in a lower $\mathrm{HAQ}^{17}$. Similarly, a French early arthritis study found that SDAI and CDAI, but not DAS28, remission at 6, 12, and 24 months gave a lower $\mathrm{HAQ}$ at 36 months compared with $\mathrm{LDA}^{18}$. The associations of 9 different composite indices, including the SDAI, CDAI, and DAS28, were shown to have high correlation to functional status, even though the proportions of patients classified in the different disease activity levels differed ${ }^{9}$. In 

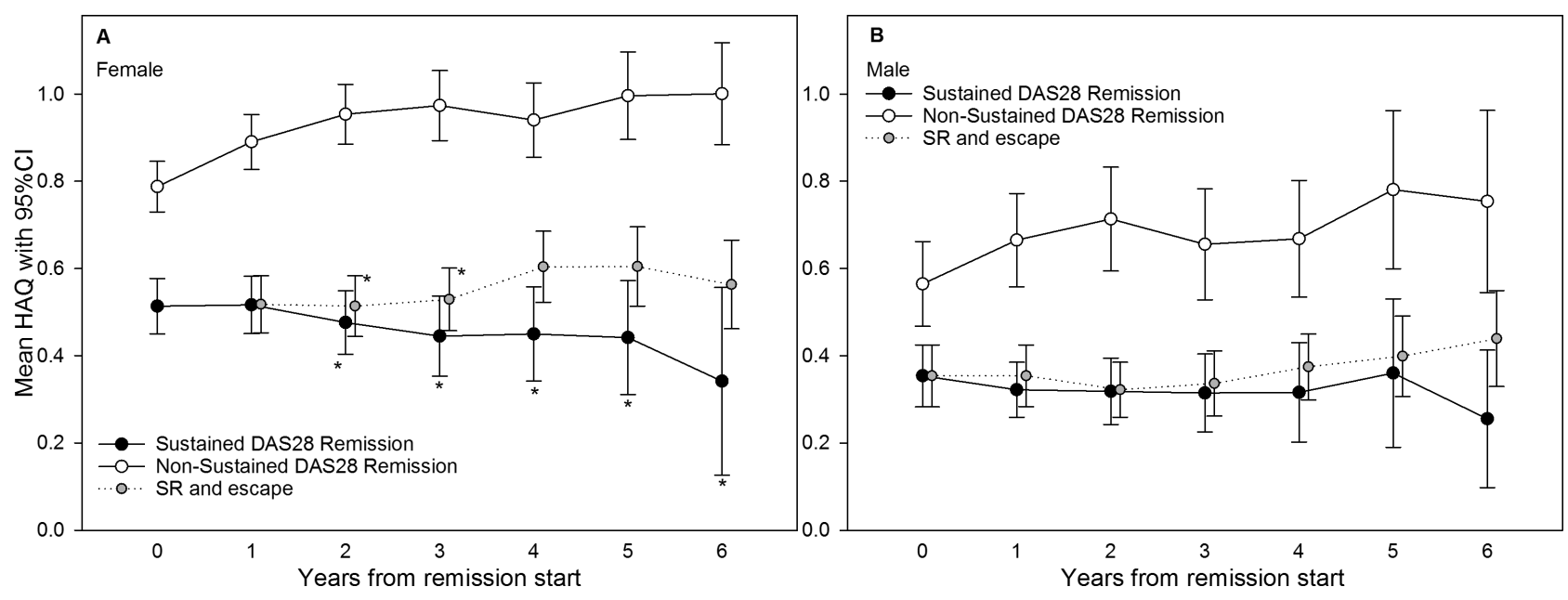

Figure 3. Course of HAQ in SR in (A) women and (B) men, separately. Year 0 is at start of SR or first remission for the NSR patient. After that, each point represents the mean HAQ during the year. For definitions, see Figure 2. Women in SR show improvement in HAQ over time, but the improvement is not significant for male patients. $*$ P $<0.05$. HAQ: Health Assessment Questionnaire; SR: sustained remission; NSR: non-sustained remission; DAS28: Disease Activity Score based on 28 joints.
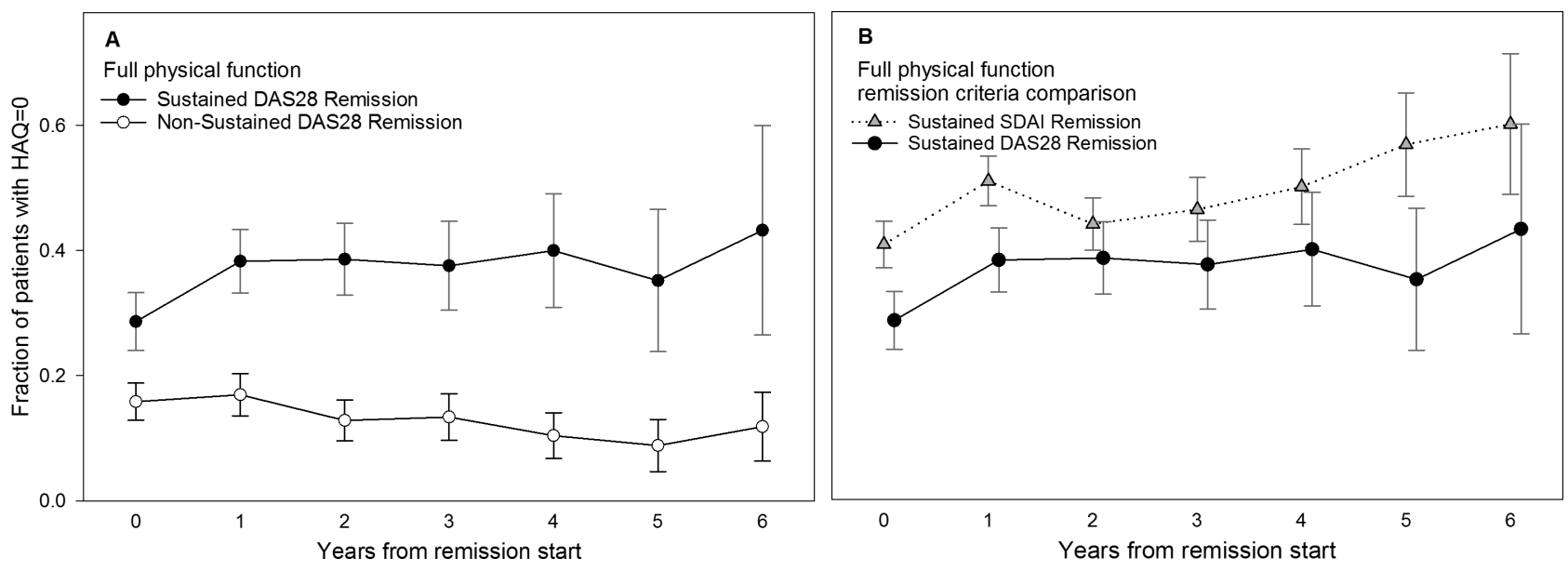

Figure 4. Full physical function (HAQ = 0). (A) Fraction of patients with full physical function defined as 0 in HAQ. The change in fraction of patients was significant in the second year of remission $(\mathrm{p}<0.05)$. (B) Fraction of patients with full physical function in the SDAI and DAS28 sustained remission compared. No difference between different remission criteria. HAQ: Health Assessment Questionnaire; SDAI: Simplified Disease Activity Index; DAS28: Disease Activity Score based on 28 joints.

our study, we have looked not at patients with LDA, but rather at patients who are only occasionally in remission, although not sustained over 6 months (NSR). Patients in DAS28 SR improved significantly in HAQ compared with those in NSR already during the first year of treatment, and the difference between the groups continued throughout the whole followup period. Moreover, the NSR group deteriorated in function over the 6-year followup. Even after escape from SR, the improvement in function largely remained for up to 8 years, whereas those retaining remission showed a further improvement in function. The pattern was similar when using the SDAI and CDAI remission criteria.

The difference between the groups is largely driven by female patients. The men show a similar trend, but the difference is not significant. Although this may partly be a power problem, RA disease activity measures have been shown to be worse in women than in men, and that the difference originates from the character of the measure rather than RA disease activity ${ }^{19,20}$. Men tend to score lower on HAQ and DAS28, and have a higher chance of being in DAS28 remission ${ }^{21}$, which might explain why the improvement is not as pronounced as in women. In the BARFOT (Bättre AntiReumatisk FarmakOTerapi) study, women and men had a similar radiologic joint destruction over 8 years despite higher disease activity and worse function in women ${ }^{22}$. 
The prevalence of full physical function $(\mathrm{HAQ}=0)$ in our cohort was $1.4 \%$ at treatment start. In a normal population, the prevalence was $74 \%$ (66-82) for people 51-55 years old and $63 \%(53-73)$ in those $56-60$ years old ${ }^{19}$. After about 50 years of age, HAQ shows an almost exponential deterioration. In contrast, patients with RA in SR show a trend toward increased prevalence of full physical function, approaching the normal population. Nevertheless, the patients with RA in our study have a much higher mean HAQ at treatment start compared with that of the normal population ${ }^{19}$.

There are limitations to our study. Although it has been previously shown ${ }^{13}$ that $98 \%$ of the patients in the SSATG registry have RA according to the 1987 ACR criteria, it is possible that some patients with other forms of inflammatory arthritis have been included. Ours is an observational study and it is possible that the patients who continue in remission simply represent patients with favorable disease progression, not successful treatment. Still, they have high HAQ (1.0) at baseline, 9 swollen joints, and a disease duration of over 10 years, but similar levels are found in the NSR group. On the other hand, there is a bias because the SR patients have a lower HAQ from the start, and we have previously shown that a low HAQ is the strongest predictor for $\mathrm{SR}^{21}$. Information on comorbidities and radiographs is not complete in our registry and we were unable to adjust for chronic conditions such as osteoarthritis and other structural damage that may influence HAQ score independently from RA disease activity. Although methodological limitations should be borne in mind when interpreting the results, observational studies remain important in offering information from daily clinical practice strengthened by their possibility for longterm followup.

Our patients had an average disease duration of almost 12 years at treatment start. Assessing disease activity in such a group is problematic because joint swelling and tenderness may be a consequence of irreversible structural damage. It has also been shown that the sensitivity to change in HAQ depends on the average disease duration of RA, with longer disease duration being less responsive ${ }^{20}$. The Boolean ACR/EULAR remission criteria for clinical trials demand a low patient's global assessment $(\leq 1 \text { on a } 0-10 \text { scale })^{7}$, this often being a limiting factor ${ }^{23,24}$. We therefore chose to use SDAI remission, also proposed by the ACR/EULAR and the clinical criteria, CDAI, as well as DAS28 remission, which is the most widely used definition ${ }^{7}$. Although the ACR/EULAR remission criteria are less relevant in our patient group with established disease, the principle of sustained versus occasional remission is important to emphasize regardless of the criteria set used.

The treat-to-target recommendations have motivated physicians toward reaching the goal of remission in patients with $\mathrm{RA}^{3}$ and the concept has contributed to new remission criteria ${ }^{7}$. However, these recommendations do not currently focus on the time perspective, i.e., the relevance of sustaining remission for prolonged time periods. In the current EULAR recommendations, SR is discussed in the context of treatment tapering, but not as a treatment target in itself. Tapering of biologic DMARD is advised only after persistent remission, and cautious reduction of conventional synthetic DMARD dose in cases of sustained longterm remission ${ }^{25}$. This might lead to fewer patients reaching SR given that only about half of the individuals in remission at a single timepoint remain in remission at a subsequent visit a year later ${ }^{26}$. Our current report underscores the importance of maintaining remission to improve the goal of full function.

Results from our present study indicate that only occasionally reaching remission might not be enough, but for improved physical function, remission should be sustained. Because the functional status is highly comparable between different remission definitions, the issue of the sustainability of disease remission might be more important than the choice of remission criteria.

\section{ACKNOWLEDGMENT}

The authors thank Jan-Åke Nilsson for his valuable and constructive criticism.

\section{REFERENCES}

1. Pincus T, Callahan LF. What is the natural history of rheumatoid arthritis? Rheum Dis Clin North Am 1993;19:123-51.

2. Smolen JS, Landewé R, Breedveld FC, Dougados M, Emery P, Gaujoux-Viala $\mathrm{C}$, et al. EULAR recommendations for the management of rheumatoid arthritis with synthetic and biological disease-modifying antirheumatic drugs. Ann Rheum Dis 2010;69:964-75.

3. Smolen JS, Aletaha D, Bijlsma JW, Breedveld FC, Boumpas D, Burmester G, et al; T2T Expert Committee. Treating rheumatoid arthritis to target: recommendations of an international task force. Ann Rheum Dis 2010;69:631-7.

4. Aletaha D, Funovits J, Breedveld FC, Sharp J, Segurado O, Smolen JS. Rheumatoid arthritis joint progression in sustained remission is determined by disease activity levels preceding the period of radiographic assessment. Arthritis Rheum 2009;60:1242-9.

5. Wolfe F, Hawley DJ. The longterm outcomes of rheumatoid arthritis: Work disability: a prospective 18 year study of 823 patients. J Rheumatol 1998;25:2108-17.

6. Wolfe F, Michaud K, Gefeller O, Choi HK. Predicting mortality in patients with rheumatoid arthritis. Arthritis Rheum 2003; 48:1530-42.

7. Felson DT, Smolen JS, Wells G, Zhang B, van Tuyl LH, Funovits J, et al. American College of Rheumatology/European League Against Rheumatism Provisional Definition of Remission in Rheumatoid Arthritis for Clinical Trials. Ann Rheum Dis 2011;70:404-13.

8. Aletaha D, Smolen JS. Joint damage in rheumatoid arthritis progresses in remission according to the Disease Activity Score in 28 joints and is driven by residual swollen joints. Arthritis Rheum 2011;63:3702-11.

9. Klarenbeek NB, Koevoets R, van der Heijde DM, Gerards AH, Ten Wolde S, Kerstens PJ, et al. Association with joint damage and physical functioning of nine composite indices and the 2011 ACR/EULAR remission criteria in rheumatoid arthritis. Ann Rheum Dis 2011;70:1815-21.

10. Geborek P, Saxne T. Clinical protocol for monitoring of targeted therapies in rheumatoid arthritis. Rheumatology 2000;39:1159-61.

Personal non-commercial use only. The Journal of Rheumatology Copyright (c) 2016. All rights reserved. 
11. Geborek P, Nitelius E, Noltorp S, Petri H, Jacobsson L, Larsson L, et al. Population based studies of biological antirheumatic drug use in southern Sweden: comparison with pharmaceutical sales. Ann Rheum Dis 2005;64:1805-7.

12. Neovius M, Simard J, Sundström A, Jacobsson L, Geborek P, Saxne T, et al; ARTIS Study Group. Generalisability of clinical registers used for drug safety and comparative effectiveness research: coverage of the Swedish Biologics Register. Ann Rheum Dis 2011;70:516-9.

13. Geborek P, Crnkic M, Petersson IF, Saxne T; South Swedish Arthritis Treatment Group. Etanercept, infliximab, and leflunomide in established rheumatoid arthritis: clinical experience using a structured follow up programme in southern Sweden. Ann Rheum Dis 2002;61:793-8.

14. Aletaha D, Ward MM, Machold KP, Nell VP, Stamm T, Smolen JS Remission and active disease in rheumatoid arthritis: defining criteria for disease activity states. Arthritis Rheum 2005; 52:2625-36

15. Kristensen LE, Kapetanovic MC, Gülfe A, Söderlin M, Saxne T, Geborek P. Predictors of response to anti-TNF therapy according to ACR and EULAR criteria in patients with established RA: results from the South Swedish Arthritis Treatment Group Register. Rheumatology 2008;47:495-9.

16. Gülfe A, Aletaha D, Saxne T, Geborek P. Disease activity level, remission and response in established rheumatoid arthritis: performance of various criteria sets in an observational cohort, treated with anti-TNF agents. BMC Musculoskelet Disord 2009;10:41.

17. Radner H, Smolen JS, Aletaha D. Remission in rheumatoid arthritis: benefit over low disease activity in patient-reported outcomes and costs. Arthritis Res Ther 2014;16:R56.

18. Ruyssen-Witrand A, Guernec G, Nigon D, Tobon G, Jamard B, Rat $\mathrm{AC}$, et al. Aiming for SDAI remission versus low disease activity at 1 year after inclusion in ESPOIR cohort is associated with better 3-year structural outcomes. Ann Rheum Dis 2015;74:1676-83.
19. Krishnan E, Sokka T, Häkkinen A, Hubert H, Hannonen P. Normative values for the Health Assessment Questionnaire Disability Index: benchmarking disability in the general population. Arthritis Rheum 2004;50:953-60.

20. Aletaha D, Ward MM. Duration of rheumatoid arthritis influences the degree of functional improvement in clinical trials. Ann Rheum Dis 2006;65:227-33.

21. Einarsson JT, Geborek P, Saxne T, Kapetanovic MC. Sustained remission in tumor necrosis factor inhibitor-treated patients with rheumatoid arthritis: a population-based cohort study. J Rheumatol 2015;42:741-8.

22. Hafström I, Bala V, Albertsson K, Forslind K, Svensson B; BARFOT study group. Joint destruction in early rheumatoid arthritis over 8 years is similar in women and men despite apparently higher disease activity and poorer function in women. Ann Rheum Dis 2011;70:709-10.

23. Kuriya B, Sun Y, Boire G, Haraoui B, Hitchon C, Pope JE, et al; Canadian Early Arthritis Cohort Study. Remission in early rheumatoid arthritis - a comparison of new ACR/EULAR remission criteria to established criteria. J Rheumatol 2012; 39:1155-8.

24. Shahouri SH, Michaud K, Mikuls TR, Caplan L, Shaver TS, Anderson JD, et al. Remission of rheumatoid arthritis in clinical practice: application of the American College of Rheumatology/European League Against Rheumatism 2011 remission criteria. Arthritis Rheum 2011;63:3204-15.

25. Smolen JS, Landewé R, Breedveld FC, Buch M, Burmester G, Dougados $\mathrm{M}$, et al. EULAR recommendations for the management of rheumatoid arthritis with synthetic and biological disease-modifying antirheumatic drugs: 2013 update. Ann Rheum Dis 2014;73:492-509.

26. Prince FH, Bykerk VP, Shadick NA, Lu B, Cui J, Frits M, et al. Sustained rheumatoid arthritis remission is uncommon in clinical practice. Arthritis Res Ther 2012;14:R68. 